\title{
The sunrise integral and elliptic polylogarithms
}

\author{
Luise Adams \\ PRISMA Cluster of Excellence, Institut für Physik, \\ Johannes Gutenberg-Universität Mainz, \\ D - 55099 Mainz, Germany \\ E-mail: ladams01@uni-mainz.de
}

\section{Christian Bogner*}

Institut für Physik, Humboldt-Universität zu Berlin,

D - 10099 Berlin, Germany

E-mail: bogner@math.hu-berlin.de

\section{Stefan Weinzierl}

PRISMA Cluster of Excellence, Institut für Physik, Johannes Gutenberg-Universität Mainz,

D - 55099 Mainz, Germany

E-mail: weinzierl@uni-mainz.de

We summarize recent computations with a class of elliptic generalizations of polylogarithms, arising from the massive sunrise integral. For the case of arbitrary masses we obtain results in two and four space-time dimensions. The iterated integral structure of our functions allows us to furthermore compute the equal mass case to arbitrary order.

Loops and Legs in Quantum Field Theory

24-29 April 2016

Leipzig, Germany

*Speaker. 


\section{Introduction}

Various methods for the symbolical computation of multi-loop Feynman integrals rely on properties of classical polylogarithms

$$
\operatorname{Li}_{n}(z)=\sum_{j=1}^{\infty} \frac{z^{j}}{j^{n}},|z|<1
$$

and their generalizations. By now the class of multiple polylogarithms $[24,25]$

$$
\operatorname{Li}_{n_{1}, \ldots n_{k}}\left(z_{1}, \ldots, z_{k}\right)=\sum_{0<j_{1}<\ldots<j_{k}} \frac{z_{1}^{j_{1}} \ldots z_{k}^{j_{k}}}{j_{1}^{n_{1}} \ldots j_{k}^{n_{k}}},\left|z_{i}\right|<1,
$$

is well-established in particle physics.

One of the advantages of these functions is their iterated integral structure. For example a classical polylogarithm of weight $n \geq 2$ can be written as

$$
\operatorname{Li}_{n}(z)=\int_{0}^{z} \frac{d x}{x} \operatorname{Li}_{n-1}(x)
$$

and similar relations hold for the generalizations.

One of the computational approaches making use of this property is the method of differential equations [29,34]. Here a Feynman integral is computed by integrating over a linear combination of other Feynman integrals. If the latter are known in terms of generalized polylogarithms and if they appear with integral kernels in an appropriate set of differential forms, then the integral over these expressions can be computed by use of relations such as eq. 1.1 and the result belongs to the same class of functions.

The computations summarized in these notes are motivated by the fact, that multiple polylogarithms are not sufficient to express every Feynman integral. We consider several cases of the massive sunrise integral, which is a famous showcase of this problem. Various classes of functions different from polylogarithms were applied to this integral in the past. More recently, the case of equal masses in two space-time dimensions was expressed with the help of an elliptic dilogarithm in [14].

We define a related class of elliptic generalizations of polylogarithms, including a generalization depending on several variables. With the help of these functions, we compute the sunrise integral in the case of arbitrary masses at two and, with the help of dimension shift relations, near four space-time dimensions. We furthermore show for the case of equal masses and two dimensions, that all orders of the Laurent expansion can be expressed with the help of our framework of functions. We provide an explicit algorithm for the computation of these orders, relying on corresponding differential equations and on the iterated integral structure of our class of functions.

\section{A class of elliptic generalizations}

We define a class of functions of variables $q, x_{1}, \ldots, x_{l}, y_{1}, \ldots, y_{l}$. They are related with polylogarithms and known elliptic generalizations. 


\subsection{Definitions}

For $l=1$ we define

$$
\operatorname{ELi}_{n ; m}(x ; y ; q)=\sum_{j=1}^{\infty} \sum_{k=1}^{\infty} \frac{x^{j}}{j^{n}} \frac{y^{k}}{k^{m}} q^{j k}
$$

and for $l>1$ we define

$$
\begin{aligned}
& \operatorname{ELi}_{n_{1}, \ldots, n_{l} ; m_{1}, \ldots, m_{l} ; 2 o_{1}, \ldots, 2 o_{l-1}}\left(x_{1}, \ldots, x_{l} ; y_{1}, \ldots, y_{l} ; q\right) \\
& =\sum_{j_{1}=1}^{\infty} \ldots \sum_{j_{l}=1}^{\infty} \sum_{k_{1}=1}^{\infty} \ldots \sum_{k_{l}=1}^{\infty} \frac{x_{1}^{j_{1}}}{j_{1}^{h_{1}}} \ldots \frac{x_{l}^{j_{l}}}{j_{l}^{h_{l}}} \frac{y_{1}^{k_{1}}}{k_{1}^{m_{1}}} \cdot \frac{y_{l}^{k_{l}}}{k_{l}^{m_{l}}} \frac{q^{j_{1} k_{1}+\ldots+j_{l} k_{l}}}{\prod_{i=1}^{l-1}\left(j_{i} k_{i}+\ldots+j_{l} k_{l}\right)^{o_{i}}}
\end{aligned}
$$

We will refer to these as ELi-functions.

By construction, this class of functions is closed under multiplication with the $(l=1)$-case $\mathrm{ELi}_{n ; m}$ and under integration over $\frac{d q}{q}$. We have

$$
\begin{gathered}
\operatorname{ELi}_{n_{1}, \ldots, n_{l} ; m_{1}, \ldots, m_{l} ; 2 o_{1}, \ldots, 2 o_{l-1}}\left(x_{1}, \ldots, x_{l} ; y_{1}, \ldots, y_{l} ; q\right) \\
=I^{o_{1}} \operatorname{ELi}_{n_{1} ; m_{1}}\left(x_{1} ; y_{1} ; q^{\prime}\right) \operatorname{ELi}_{n_{2}, \ldots, n_{l} ; m_{2}, \ldots, m_{l} ; 2 o_{2}, \ldots, 2 o_{l-1}}\left(x_{2}, \ldots, x_{l} ; y_{2}, \ldots, y_{l} ; q^{\prime}\right)
\end{gathered}
$$

where $I^{o_{i}}$ denotes the $o_{i}$-fold integration

$$
I^{o_{i}}=\int_{0}^{q} \frac{d q_{1}}{q_{1}} \int_{0}^{q_{1}} \frac{d q_{2}}{q_{2}} \ldots \int_{0}^{q_{o_{i}-2}} \frac{d q_{o_{i}-1}}{q_{o_{i}-1}} \int_{0}^{q_{o_{i}-1}} \frac{d q^{\prime}}{q^{\prime}} \text { for } o_{i}>0
$$

and $I^{0}=1$.

Combining the above ELi-functions, we furthermore define a class which we will refer to as E-functions by

$$
\mathrm{E}_{n ; m}(x ; y ; q)=d_{n, m}\left(\frac{1}{2} \operatorname{Li}_{n}(x)+c_{n, m} \frac{1}{2} \operatorname{Li}_{n}\left(x^{-1}\right)+\operatorname{ELi}_{n ; m}(x ; y ; q)+c_{n, m} \operatorname{ELi}_{n ; m}\left(x^{-1} ; y^{-1} ; q\right)\right)
$$

where $c_{n, m}=-1, d_{n, m}=-i$ for even $n+m$ and $c_{n, m}=1, d_{n, m}=1$ for odd $n+m$. We furthermore define

$$
\begin{gathered}
\mathrm{E}_{n_{1}, \ldots, n_{l} ; m_{1}, \ldots, m_{l} ; 2 o_{1}, \ldots, 2 o_{l-1}}\left(x_{1}, \ldots, x_{l} ; y_{1}, \ldots, y_{l} ; q\right) \\
=I^{o_{1}}\left(\mathrm{E}_{n_{1} ; m_{1}}\left(x_{1} ; y_{1} ; q^{\prime}\right)-\mathrm{E}_{n_{1} ; m_{1}}\left(x_{1} ; y_{1} ; 0\right)\right) \mathrm{ELi}_{n_{2}, \ldots, n_{l} ; m_{2}, \ldots, m_{l} ; 2 o_{2}, \ldots, 2 o_{l-1}}\left(x_{2}, \ldots, x_{l} ; y_{2}, \ldots, y_{l} ; q^{\prime}\right) .
\end{gathered}
$$

Our results for the sunrise integral, discussed below, will be expressed in terms of E-functions and multiple polylogarithms.

\subsection{Relations with known functions}

In the case of all $o$-indices being zero, the ELi-functions are products of the $(l=1)$-case:

$$
\operatorname{ELi}_{n_{1}, \ldots, n_{l} ; m_{1}, \ldots, m_{l} ; 0, \ldots, 0}\left(x_{1}, \ldots, x_{l} ; y_{1}, \ldots, y_{l} ; q\right)=\prod_{i=1}^{l} \operatorname{ELi}_{n_{i} ; m_{i}}\left(x_{i} ; y_{i} ; q\right) .
$$


For $q=1$ the latter is furthermore just a product of polylogarithms due to

$$
\operatorname{ELi}_{n ; m}(x ; y ; q)=\sum_{k=1}^{\infty} \frac{y^{k}}{k^{m}} \operatorname{Li}_{n}\left(q^{k} x\right) \text { and } \operatorname{ELi}_{n ; m}(x ; y ; 1)=\operatorname{Li}_{n}(x) \operatorname{Li}_{m}(y) .
$$

More notably, the E-functions are related to known versions of elliptic polylogarithms. Let us briefly recall a basic principle behind such functions. We consider a lattice of points $L=\mathbb{Z}+\tau \mathbb{Z}$ where $\tau \in \mathbb{C}$ with $\operatorname{Im}(\tau)>0$. A function of $x \in \mathbb{C}$ is called elliptic with respect to $L$ if it is periodic under $x \rightarrow x+\lambda$ with $\lambda \in L$. For a function $F$ of $z=e^{2 \pi i x} \in \mathbb{C}^{\star}$ this condition translates to

$$
F(z)=F(z q) \text { for } q=e^{2 \pi i \lambda}, \lambda \in L
$$

This concept was first applied to define an elliptic dilogarithm in [11]. Generalizations were introduced in $[9,23,31,40]$.

In [17] elliptic polylogarithms are defined as coefficients of the regular part of the Laurent expansion around $\alpha=0$ of functions

$$
E_{m}(z ; u ; q)=\sum_{n \in \mathbb{Z}} u^{n} \operatorname{Li}_{m}\left(q^{n} z\right)
$$

with $u=e^{2 \pi i \alpha}$. The latter functions are related to the above functions $\mathrm{E}_{n ; m}(x ; y ; q)$. We have for example

$$
\begin{aligned}
\mathrm{E}_{2 ; 0}(x ; y ; q)= & \frac{1}{i}\left(E_{2}(x ; y ; q)-\frac{1}{2} \frac{1+y}{1-y} \zeta(2)-\frac{1}{4} \frac{1+y}{1-y} \ln ^{2}(-x)\right. \\
& \left.-\frac{y}{(1-y)^{2}} \ln (-x) \ln (q)-\frac{1}{2} \frac{y(1+y)}{(1-y)^{3}} \ln ^{2}(q)\right) .
\end{aligned}
$$

The functions $\mathrm{E}_{n ; m}(x ; y ; q)$ can furthermore be understood as generalizations of Clausen- and Glaisherfunctions, which are defined by

$$
\mathrm{Cl}_{n}(\varphi)=\frac{1}{2 i}\left(\mathrm{Li}_{n}\left(e^{i \varphi}\right)-\mathrm{Li}_{n}\left(e^{-i \varphi}\right)\right), \mathrm{Gi}_{n}(\varphi)=\frac{1}{2}\left(\operatorname{Li}_{n}\left(e^{i \varphi}\right)+\mathrm{Li}_{n}\left(e^{-i \varphi}\right)\right)
$$

for even $n$ and by

$$
\mathrm{Cl}_{n}(\varphi)=\frac{1}{2}\left(\mathrm{Li}_{n}\left(e^{i \varphi}\right)+\mathrm{Li}_{n}\left(e^{-i \varphi}\right)\right), \mathrm{Gl}_{n}(\varphi)=\frac{1}{2 i}\left(\operatorname{Li}_{n}\left(e^{i \varphi}\right)-\mathrm{Li}_{n}\left(e^{-i \varphi}\right)\right)
$$

for odd $n$. We have

$$
\lim _{q \rightarrow 0} \mathrm{E}_{n ; m}\left(e^{i \varphi} ; y ; q\right)=\mathrm{Cl}_{n}(\varphi)
$$

for $m$ being zero or even and

$$
\lim _{q \rightarrow 0} \mathrm{E}_{n ; m}\left(e^{i \varphi} ; y ; q\right)=\mathrm{Gl}_{n}(\varphi)
$$

for $m$ being odd. 


\section{Cases of the massive sunrise integral}

The massive sunrise integral

$$
S(D, t)=\int \frac{d^{D} k_{1} d^{D} k_{2}}{\left(i \pi^{D / 2}\right)^{2}} \frac{1}{\left(-k_{1}^{2}+m_{1}^{2}\right)\left(-k_{2}^{2}+m_{2}^{2}\right)\left(-\left(p-k_{1}-k_{2}\right)^{2}+m_{3}^{2}\right)},
$$

which in various versions was considered by many authors $[5-8,10,15,16,18-22,26-28,30,33$, $35,36,39$ ], is a showcase for the mentioned problem, that there are Feynman integrals which can not be expressed entirely in terms of multiple polylogarithms. For arbitrary masses and arbitrary dimension $D$, the integral was computed in [10] in terms of Lauricella functions of type C. The fact that none of the existing techniques provides a way to expand these functions in terms of multiple polylogarithms so far may be seen as a confirmation of the mentioned problem.

With respect to the variable $t=p^{2}$ which we consider in the region $t \leq 0$, the integral $S(D, t)$ satisfies a differential equation

$$
L_{4} S(D, t)=T(D, t) .
$$

Here $L_{4}$ is a differential operator of fourth order and the inhomogeneous part $T(D, t)$ is a combination of tadpole integrals, all of whose coefficients are polynomials in $m_{1}^{2}, m_{2}^{2}, m_{3}^{2}, t, D$. In the following we will consider coefficients in the Laurent series of $S(D, t)$, satisfying differential equations of fourth or lower order. These coefficients will arise from the expansion at $D=2$ and at $D=4$ dimensions:

$$
\begin{aligned}
& S(2-2 \varepsilon, t)=S^{(0)}(2, t)+S^{(1)}(2, t) \varepsilon+\mathscr{O}\left(\varepsilon^{2}\right), \\
& S(4-2 \varepsilon, t)=S^{(-2)}(4, t) \varepsilon^{-2}+S^{(-1)}(4, t) \varepsilon^{-1}+S^{(0)}(4, t)+\mathscr{O}(\varepsilon) .
\end{aligned}
$$

\subsection{The case of $D=2$ dimensions}

The case of exactly $D=2$ dimensions is a good starting point for several reasons. Firstly, the Feynman integral is finite here. The Laurent expansion in eq. 3.1 begins with $S^{(0)}(2, t)$ which satisfies a differential equation [32]

$$
L_{2} S^{(0)}(2, t)=P(t)
$$

where $L_{2}$ is a second order differential operator with respect to $t$ whose coefficients are polynomials in the squared masses and $t$. The inhomogeneous part $P(t)$ furthermore involves logarithms of the squared masses.

Secondly, if we write the Feynman integral in terms of Feynman parameters, the first Symanzik polynomial drops out in $D=2$ dimensions and the integrand only involves the second one, which reads

$$
\mathscr{F}=-x_{1} x_{2} x_{3} t+\left(x_{1} m_{1}^{2}+x_{2} m_{2}^{2}+x_{3} m_{3}^{2}\right)\left(x_{1} x_{2}+x_{2} x_{3}+x_{1} x_{3}\right) .
$$

Even though we do not attempt to integrate out the Feynman parameters, this polynomial plays an important role in our computations. The zero set of this polynomial intersects the domain of the Feynman parametric integral at three points in its corners. By choosing one of these points as 
the origin, we obtain an elliptic curve defined by $\mathscr{F}$. The corresponding Weierstrass normal form defines three zeros $e_{1}, e_{2}, e_{3}$ of the cubical equation. Using these as integration boundaries, one canonically defines two period integrals $\psi_{1}, \psi_{2}$ of the elliptic curve. These evaluate to

$$
\psi_{1}=\frac{4}{\tilde{D}^{\frac{1}{4}}} K(k), \psi_{2}=\frac{4 i}{\tilde{D}^{\frac{1}{4}}} K\left(k^{\prime}\right)
$$

where

$$
K(x)=\int_{0}^{1} d t \frac{1}{\sqrt{\left(1-t^{2}\right)\left(1-x^{2} t^{2}\right)}}
$$

is the complete elliptic integral of first kind and where

$$
k=\sqrt{\frac{e_{3}-e_{2}}{e_{1}-e_{2}}}, k^{\prime}=\sqrt{1-k^{2}}=\sqrt{\frac{e_{1}-e_{3}}{e_{1}-e_{3}}}
$$

and

$$
\tilde{D}=\left(t-\left(m_{1}+m_{2}-m_{3}\right)^{2}\right)\left(t-\left(m_{1}-m_{2}+m_{3}\right)^{2}\right)\left(t-\left(-m_{1}+m_{2}+m_{3}\right)^{2}\right)\left(t-\left(m_{1}+m_{2}+m_{3}\right)^{2}\right) .
$$

As $\psi_{1}$ and $\psi_{2}$ are solutions of the homogeneous equation $L_{2} S^{(0)}(2, t)=0$, the special solution of the inhomogeneous eq. 3.3 can be constructed by classical variation of constants as an integral over a certain combination of the homogeneous solutions. In this way, we obtain the full solution involving an integral over complete elliptic integrals [1].

However, we find [2] that the solution can be written alternatively as

$$
\begin{aligned}
S^{(0)}(2, t) & =\frac{\psi_{1}(q)}{\pi} E^{(0)}, \\
E^{(0)} & =\sum_{i=1}^{3} \mathrm{E}_{2 ; 0}\left(w_{i}(q) ;-1 ;-q\right)
\end{aligned}
$$

where $\mathrm{E}_{2 ; 0}$ is one of the E-functions. The dependence on $t$ is now given in terms of $q$ which we define as $q=e^{\pi i \frac{\psi_{2}(t)}{\psi_{1}(t)}}$ in terms of the period integrals of our elliptic curve. The three arguments $w_{1}$, $w_{2}, w_{3}$ are obtained explicitly from the mentioned intersection points by transformations on the elliptic curve.

\subsection{Higher orders and four dimensions}

Computing higher orders in the Laurent expansion is interesting for several reasons. First of all, we obtain a result for the four-dimensional case in this way. While the pole terms of eq. 3.2 were already known, we obtain [3] the coefficient $S^{(0)}(4, t)$ in terms of $S^{(0)}(2, t), S^{(1)}(2, t)$, $\frac{\partial}{\partial m_{i}^{2}} S^{(0)}(2, t), \frac{\partial}{\partial m_{i}^{2}} S^{(1)}(2, t), i=1,2,3$ by use of dimension shift relations [37,38]. As $S^{(0)}(2, t)$ is given by eq. 3.4 , the missing ingredient here is $S^{(1)}(2, t)$. This coefficient satisfies a fourth order differential equation

$$
L_{1, a} L_{1, b} L_{2} S^{(1)}(2, t)=I_{1}(t)
$$

where the inhomogeneous part $I_{1}(t)$ involves a polynomial of the squared masses and $t$, logarithms of the squared masses and the coefficient $S^{(0)}(2, t)$. The differential operator factorizes into two 
operators of first order $L_{1, a}, L_{1, b}$ and an operator $L_{2}$ of second order which we already know from eq. 3.3. Due to this factorization, we obtain the second order differential equation

$$
L_{2} S^{(1)}(2, t)=I_{2}(t)
$$

where the only difference to eq. 3.3 is a more complicated inhomogeneous part $I_{2}(t)$. Applying variation of constants as above, we obtain an explicit result for $S^{(1)}(2, t)$ which we express in terms of E-functions. We arrive at [3]

$$
\begin{aligned}
S^{(1)}(2, t)= & \frac{\psi_{1}(q)}{\pi} E^{(1)}, \\
E^{(1)}= & \left(\sum_{j=1}^{3}\left(\mathrm{E}_{1 ; 0}\left(w_{j} ; 1 ;-q\right)-\frac{1}{3} E_{1 ; 0}\left(w_{j} ;-1 ;-q\right)\right)-\frac{2}{3} \sum_{j=1}^{3} \ln \left(\frac{m_{j}^{2}}{\mu^{2}}\right)\right. \\
& \left.-6 \mathrm{E}_{1 ; 0}(-1 ; 1 ;-q)\right) E^{(0)}+E_{R}^{(1)}
\end{aligned}
$$

where $E_{R}^{(1)}$ is a linear combination of the functions $\mathrm{Li}_{2}, \mathrm{Li}_{3}, \mathrm{Li}_{2,1}, \mathrm{E}_{3 ; 1}$ and $\mathrm{E}_{0,1 ;-2,0 ; 4}$ and where $\mathrm{E}^{(0)}$ is defined in eq. 3.5. With the help of this result, one obtains the coefficient $S^{(0)}(4, t)$ of the sunrise integral around four dimensions.

The other reason for computing even higher orders in the Laurent series is our interest in the functions appearing there. Now we consider the case of equal masses $m=m_{1}=m_{2}=m_{3}$. As a first step, we define

$$
\tilde{S}(2-2 \varepsilon, t)=\sum_{j=0}^{\infty} \varepsilon^{j} \tilde{S}^{(j)}(2, t)
$$

by

$$
S(2-2 \varepsilon, t)=\Gamma(1+\varepsilon)^{2}\left(\frac{3 \mu^{4} \sqrt{t}}{m\left(t-m^{2}\right)\left(t-9 m^{2}\right)}\right)^{\varepsilon} \tilde{S}(2-2 \varepsilon, t) .
$$

The differential equations simplify for $\tilde{S}$ and allow us to recursively express any coefficient in the Laurent series as

$$
\tilde{S}^{(j)}=-\frac{\psi_{1}}{\pi} \int_{q_{0}}^{q} \frac{d q_{1}}{q_{1}} \int_{q_{0}}^{q_{1}} \frac{d q_{2}}{q_{2}}\left(a_{j}+b \tilde{S}^{(j-2)}\right) \text { for } j \geq 2 .
$$

We can show that all functions $a_{j}$ and the $j$-independent function $b$ in this equation can be expressed as products of ELi-functions. The lowest coefficients $\tilde{S}^{(0)}, \tilde{S}^{(1)}$ are immediately obtained from our previous results in terms of E- or ELi-functions. Therefore, due to the properties of our classes of functions discussed in section 2.1, all coefficients $\tilde{S}^{(j)}$ can be expressed in terms of E-functions, together with classical and multiple polylogarithms. As a consequence, the same is true for all orders of $S(2-2 \varepsilon, t)[4]$.

\section{Conclusions}

By use of the class of functions defined in section 2.1 we have computed the sunrise integral to order $\mathscr{O}(\varepsilon)$ in two dimensions and to order $\mathscr{O}\left(\varepsilon^{0}\right)$ in four dimensions. For the case of equal masses and two dimensions, we presented an algorithm to compute all orders. The iterated integral structure of our class of functions has shown to be useful in the systematic use of the method of differential equations. It therefore suggests itself to be used in future computations of further integrals, beyond multiple polylogarithms and beyond the sunrise. 


\section{References}

[1] L. Adams, C. Bogner, and S. Weinzierl, The two-loop sunrise graph with arbitrary masses, J. Math. Phys. 54, 052303 (2013), arXiv:1302.7004 [hep-ph].

[2] L Adams, C Bogner, and S Weinzierl, The two-loop sunrise graph in two space-time dimensions with arbitrary masses in terms of elliptic dilogarithms, J.Math.Phys. 55 (2014) 10, 102301,arXiv:1405.5640 [hep-ph].

[3] L. Adams, C. Bogner, and S. Weinzierl,The two-loop sunrise integral around four space-time dimensions and generalisations of the Clausen and Glaisher functions towards the elliptic case, J.Math. Phys. 56 (2015) no.7, 072303, arXiv:1504.03255 [hep-ph].

[4] L. Adams, C. Bogner, and S. Weinzierl, The iterated structure of the all-order result for the two-loop sunrise integral, J.Math.Phys. 57 (2016) no.3, 032304, arXiv:1512.05630 [hep-ph].

[5] D.H. Bailey, J.M. Borwein, D. Broadhurst, and M.L. Glasser, Elliptic integral evaluations of Bessel moments, J.Phys. A41 (2008) 205203, arXiv:0801.0891.

[6] S. Bauberger, M. Böhm, G. Weiglein, F.A. Berends and M. Buza, Calculation of two-loop self-energies in the electroweak Standard Model, Nucl. Phys. Proc. Suppl. 37B, 95 (1994), arXiv:hep-ph/9406404.

[7] S. Bauberger, F.A. Berends, M. Böhm and M. Buza, Analytical and numerical methods for massive two loop selfenergy diagrams, Nucl. Phys. B434, 383 (1995), arXiv:hep-ph/9409388.

[8] S. Bauberger and M. Böhm, Simple one-dimensional integral representations for two loop selfenergies: The Master diagram, Nucl. Phys. B445, 25 (1995), arXiv:hep-ph/9501201.

[9] A. Beilinson and A. Levin, Elliptic Polylogarithms, Proceedings of Symposia in Pure Mathematics, Vol. 55 (1994), Part 2, pp. 126-196.

[10] F.A. Berends, M. Buza, M. Böhm and R Scharf, Closed expressions for specific massive multiloop selfenergy integrals, Z.Phys. C63, 227 (1994).

[11] S. Bloch, Higher regulators, algebraic $K$-theory and zeta-functions of elliptic curves, lecture notes, U.C.Irvine, 1977.

[12] S. Bloch, M. Kerr and P. Vanhove, A Feynman integral via higher normal functions, Compos. Math. 151 (2015) 2329-2375, arXiv:1406.2664.

[13] S. Bloch, M. Kerr and P. Vanhove, Closed expressions for specific massive multiloop selfenergy integrals, arXiv:1601.08181 [hep-th].

[14] S. Bloch and P. Vanhove, The elliptic dilogarithm for the sunset graph, Journal of Number Theory 148 (2015) pp. 328-364, arXiv:1309.5865 [hep-th].

[15] D. Broadhurst, Elliptic integral evaluation of a Bessel moment by contour integration of a lattice Green function, arXiv:0801.4813.

[16] D.J. Broadhurst, J. Fleischer and O. Tarasov, Two-loop two-point functions with masses: asymptotic expansions and Taylor series, in any dimension, Z.Phys. C60, 287 (1993), arXiv:hep-ph/9304303.

[17] F. Brown and A. Levin, Multiple elliptic polylogarithms, arXiv:1110.6917[math.NT].

[18] M. Caffo, H. Czyz, S. Laporta and E. Remiddi, Master equations for master amplitudes, Nuovo Cim. A111, 365 (1998), arXiv:hep-th/9805118. 
[19] M. Caffo, H. Czyz, M. Gunia and E. Remiddi, BOKASUN: A Fast and precise numerical program to calculate the Master Integrals of the two-loop sunrise diagrams, Comput. Phys. Commun. 180, 427 (2009), arXiv:0807.1959.

[20] M. Caffo, H. Czyz and E. Remiddi, Numerical evaluation of the general massive 2 loop sunrise selfmass master integrals from differential equations, Nucl. Phys. B634, 309 (2002), arXiv:hep-ph/0203256.

[21] A.I. Davydychev and B. Delbourgo, A Geometrical angle on Feynman integrals, J. Math. Phys. 39, 4299 (1998), arXiv:hep-th/9709216.

[22] A.I. Davydychev and V.A. Smirnov, Threshold expansion of the sunset diagram, Nucl.Phys. B554 (1999) 391-414.

[23] H. Gangl and D. Zagier, Classical and Elliptic Polylogarithms and Special Values of L-Series, in: The arithmetic and geometry of algebraic cycles Banff, AB, 1998, NATO Science Series C, Mathematical and Physical Sciences, vol 548, 561-615; Kluwer Academic Publishers, Dordrecht, 2000.

[24] A.B. Goncharov, Multiple polylogarithms, cyclotomy and modular complexes, Math Res. Letters 5, (1998) 497-516, arXiv:1105.2076 [math.AG].

[25] A.B. Goncharov, Multiple polylogarithms and mixed Tate motives, arXiv:math.AG/0103059.

[26] S. Groote, J.G. Körner, and A.A. Pivovarov, On the evaluation of a certain class of Feynman diagrams in $x$-space: Sunrise-type topologies at any loop order, Annals Phys. 322, 2374 (2007), arXiv:hep-ph/0506286.

[27] S. Groote, J. Körner, and A.A. Pivovarov, A Numerical Test of Differential Equations for One- and Two-Loop sunrise Diagrams using Configuration Space Techniques, Eur.Phys.J. C72, 2085 (2012), arXiv:1204.0694.

[28] M.Yu. Kalmykov and B.A. Kniehl, Towards all-order Laurent expansion of generalized hypergeometric functions around rational values of parameters, Nucl. Phys. B809, 365 (2009), arXiv:0807.0567.

[29] A.V. Kotikov, Differential equations method: New technique for massive Feynman diagrams calculation, Phys. Lett. B254 (1991), 158-164.

[30] S. Laporta and E. Remiddi, Analytic treatment of the two loop equal mass sunrise graph, Nucl. Phys. B704, 349 (2005), hep-ph/0406160.

[31] A. Levin, Elliptic polylogarithms: an analytic theory, Compositio Math. 106 (1997), 267-282.

[32] S. Müller-Stach, S. Weinzierl and R. Zayadeh, A Second-Order Differential Equation for the Two-Loop Sunrise Graph with Arbitrary Masses, Commun. Num. Theor. Phys. 6 (2012) 203-222, arXiv:1112.4360 [hep-ph].

[33] S. Pozzorini and E. Remiddi, Precise numerical evaluation of the two loop sunrise graph master integrals in the equal mass case, Comput. Phys. Commun. 175, 381 (2006), arXiv:hep-ph/0505041.

[34] E. Remiddi, Differential equations for Feynman graph amplitudes, Nuovo Cim. A110 (1997), 1435. Nucl. Phys. B580 (2000), 485.

[35] E. Remiddi and L. Tancredi, Schouten identities for Feynman graph amplitudes; The Master Integrals for the two-loop massive sunrise graph, Nucl.Phys. B880, 343 (2014), arXiv:1311.3342.

[36] E. Remiddi and L. Tancredi, Differential equations and dispersion relations for Feynman amplitudes. The two-loop massive sunrise and the kite integral, arXiv:1602.01481 [hep-ph]. 
[37] O.V. Tarasov, Connection between Feynman integrals having different values of the space-time dimension, Phys. Rev. D54, 6479 (1996), hep-th/9606018.

[38] O.V. Tarasov, Generalized recurrence relations for two loop propagator integrals with arbitrary masses, Nucl. Phys. B502, 455 (1997), hep-ph/9703319.

[39] N.I. Ussyukina and A.I. Davydychev, An approach to the evaluation of three-and four-point ladder diagrams, Phys. Lett. B298, 363 (1993).

[40] D. Zagier, The Bloch-Wigner-Ramakrishnan polylogarithm function, Math.Ann 286, 613-624 (1990). 